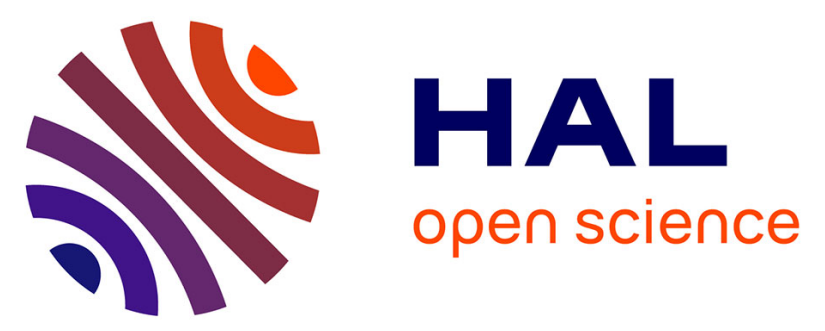

\title{
La représentation des causes de l'état de santé par les infirmiers et les points de vue envisagés des patients et des aidants naturels : une approche socionormative
}

Daniel Gilibert, I. Banovic

\section{- To cite this version:}

Daniel Gilibert, I. Banovic. La représentation des causes de l'état de santé par les infirmiers et les points de vue envisagés des patients et des aidants naturels : une approche socionormative. Psychologie du travail et des organisations, 2008, 14, pp.55-76. hal-00568207

\section{HAL Id: hal-00568207 \\ https://u-bourgogne.hal.science/hal-00568207}

Submitted on 8 Apr 2011

HAL is a multi-disciplinary open access archive for the deposit and dissemination of scientific research documents, whether they are published or not. The documents may come from teaching and research institutions in France or abroad, or from public or private research centers.
L'archive ouverte pluridisciplinaire HAL, est destinée au dépôt et à la diffusion de documents scientifiques de niveau recherche, publiés ou non, émanant des établissements d'enseignement et de recherche français ou étrangers, des laboratoires publics ou privés. 


\section{La représentation des causes de l'état de santé par les infirmiers et les points de vue envisagés des patients et des aidants naturels : une approche socionormative.}

\section{Nurses' representation of the determinants of the state of health and the points of view attributed to patients and caregivers: a socionormative approach.}

Daniel Gilibert et Ingrid Banovic

Daniel.Gilibert@u-bourgogne.fr

Daniel GILIBERT, Université de Bourgogne

Pôle AAFE, SPMS, EA4180 B.P. 26513, 21065 Dijon Cédex France

\section{Résumé}

Cette étude porte sur la représentation des déterminants de l'état de santé lors de prises en charges thérapeutiques. Nous décrivons ici la représentation d'infirmiers hospitaliers expérimentés $(n=44)$ et les différences de points de vue qu'ils envisagent avec leurs usagers. En se basant sur la finalité du soin et l'asymétrique existante dans la relation soignant-soigné, nous faisons d'une part l'hypothèse que ces infirmiers exhiberont une représentation de l'état de santé centrée sur le soin à apporter : les déterminants de l'état de santé devant être perçus comme ayant un impact général sur la vie des patients tout en étant remédiables, c'est à dire susceptibles d'évoluer et contrôlables par l'aide apportée. D'autre part, on peut s'attendre à ce qu'ils attribuent au patient une position et une attitude passive : les déterminants de son état de santé devant être perçus comme incontrôlables par lui. Les résultats et l'analyse des représentations qu'ils rapportent comme étant celles du patient et de sa famille semblent justifier la perception et le rôle soignant : les représentations supposées de ces derniers seraient convergentes mais moins centrées sur le problème de santé et minimiseraient l'implication du patient, notamment en cas d'évolution défavorable. Ils sont discutés au regard du Health Belief Model et de la norme sociale d'internalité, ainsi que des pratiques professionnelles en milieu hospitalier.

\section{Abstract}

This study examines the representation of the determining factors of patients' states of health in therapeutic care situations. We describe the average representation of experienced hospital nurses $(n=44)$ and the differences which they consider to exist between their own and their patients' points of view. Taking as our starting point the purpose of the care provided and the asymmetry of the carer/patient relationship, we hypothesize, on the one hand, that nurses will have a representation of their patients' state of health which is based on the care to be provided, with the factors determining the state of health being necessarily perceived as having a general impact on patients' lives while still being remediable, i.e. capable of changing and being controlled by the provided care. On the other hand, we expect them to attribute a passive position and attitude to their patients, with the factors determining their state of health being necessarily perceived as being out of their own control. The results and the analysis of the representations reported as being those of the patients and their families seem to justify this perception and role of carer: the supposed representations of the latter would appear to be convergent but less focused on health problems and would tend to minimize the patient's involvement, in particular when the state of health declines. The results are discussed with reference to the Health Belief Model and the social norm of internality as well as the professional practices observed in hospital environments.

Mots-clefs: Dimensions Causales - Norme Sociale-Maladie - Soignants - Health Belief Model Keywords: Causal dimensions - Social norms - Illness -Caregivers -Health Belief Model 


\section{Introduction}

Les recherches en psychologie de la santé abordent la question de la prise en charge thérapeutique comme conditionnée par un système où les points de vue de différents acteurs interviennent et interagissent. Elles traitent parfois des interactions entre soignants et patients et, depuis peu, de l'implication des aidants naturels. Cette perspective dépasse la seule prise en compte de la représentation médicale de la maladie et de son traitement. Elle vise à prendre en compte les représentations plus «naïves» du patient et de ses aidants naturels, intervenant également dans le déroulement de la prise en charge thérapeutique. Nécessairement en interaction dans la mise en place des comportements de soins, les représentations des différents acteurs semblent coexister, s'influencer mutuellement et peuvent parfois interférer les unes avec les autres. On peut alors s'interroger sur la façon dont ces acteurs s'accordent en général sur une représentation commune de la maladie, de son origine et des actions de soins à mettre en place. Si traiter une telle question demeure complexe et ambitieux, on peut tout au moins s'intéresser, dans un premier temps, à la façon dont les soignants se représentent les déterminants de l'évolution thérapeutique en relation avec les points de vue des autres acteurs concernés (patients, familles). La prise en charge de la maladie étant largement conditionnée par le savoir médical des soignants et leur préséance dans le choix des soins, on peut alors se demander: $1 /$ quels sont, du point de vue des soignants, les déterminants de l'état de santé du patient, 2/ s'ils s'attendent à ce que leur représentation soit partagée par le patient et les aidants naturels (la famille), 2/ si leur analyse causale de la maladie et les points de vue attendus de ces usagers seront les mêmes selon que l'état de santé s'améliore ou non. Cette contribution concerne donc la représentation que des soignants ont de la maladie et de la prise en charge thérapeutique en fonction des trois points de vue susceptibles dintervenir dans le système de soins: leur point de vue de soignant, celui du soigné et celui de son aidant naturel.

En partant du constat de l'asymétrie existante dans la relation du soignant avec le patient, dont la santé est supposée dépendante du soin prodigué, des hypothèses peuvent être posées : 1/ en ce qui concerne la perception que les soignants ont des déterminants de l'état de santé : ces déterminants devraient être perçus comme décisifs, susceptibles d'évoluer, dépendants de l'aide reçue plutôt que du contrôle du patient, 2/ en ce qui concerne le point de vue du malade (et de sa famille) qui serait supposé moins centré sur le problème : il sous-estimerait le fait que ces déterminants sont décisifs et remédiables, et plus passif : il surestimerait l'intervention d'éléments extérieurs, incontrôlables par lui-même (en accord avec les travaux sur les points de vue attendus dans les relations asymétriques) 3/ enfin, lorsque l'état de santé s'est détérioré, les soignants devraient avoir une perception moins optimiste des déterminants de l'état de santé et évoquer des points de vue plus fortement divergents. Afin de servir cet objectif de recherche, nous proposons dans un premier temps de passer rapidement en revue les travaux soulignant l'importance d'une approche des relations soignant-patient-aidants naturels. Dans un second temps, nous rappellerons quelques travaux montrant que les 
explications, ainsi que les points de vue envisagés pour autrui, sont dépendants des rôles professionnels et de l'asymétrie existant dans la relation.

\section{L'intervention des aidants naturels, des patients et des soignants dans la prise en charge thérapeutique}

En posant la question de la prise en charge thérapeutique comme résultant d'un système composé des interactions entre patient, soignant et aidant naturel, les travaux en psychologie de la santé tentent d'éclairer le rôle spécifique de ces acteurs dans l'observance et l'éducation thérapeutique(1). Ils amènent à s'intéresser aux interactions possibles entre soignant et patient (EIder, Ayala, Harris, 1999; Fischer, Tarquinio, 2006; Goldring, Taylor, Kemeny, Anton, 2002; Hessen-Klemens \& Lapinska, 1984). Ainsi, concernant l'observance thérapeutique, trois facteurs semblent importants: (a) le soutien que le patient peut trouver dans son entourage familial et personnel, (b) les attentes que le patient peut avoir quant à ses possibilités de guérison, l'incitant à mettre en œuvre des comportements adéquats, et enfin (c) la qualité de la relation thérapeutique avec le soignant.

Concernant tout d'abord la participation des aidants naturels, un élément illustratif de l'importance d'une approche «interactive », est que le soutien, ressenti par le malade en provenance de son entourage, semble intervenir sur les conséquences de la maladie et du traitement (Dew, Goyocoolea, Harris, Lee, Zomak, Dunbar-Jacob \& Rotondi, 2004; Pibernik-Okanovic, Prasek, Poljicanin-Filipovic, Pavlic-Renar, Metelko, 2004; van Wijngaarden, Schene, \& Koeter, 2004).

Le système de croyances et de représentations des patients serait déterminant dans la compréhension et la mise en œuvre de comportements d'observance thérapeutique. A ce sujet, le Health Relief Model (Rosenstock, 1974; Rosenstock, Strecher \& Becker, 1988) souligne l'importance des évaluations faites par les patients concernant l'utilité des prescriptions et du respect du traitement médical. Ce modèle insiste plus précisément sur l'importance du sentiment d'efficacité personnelle du patient, c'est-à-dire son sentiment de pouvoir contrôler, par des efforts personnels, l'évolution de sa maladie (Goldring, Taylor, Kemeny \& Anton 2002; Gao, Nau, Rosenbluth, Scott \& Woodward, 2000; Bradley, 1995; Home \& Weinman, 1999). La façon dont les patients conçoivent l'origine de ce qui leur arrive serait une variable importante. Ainsi le «Locus of control» différencierait les individus en deux catégories extrêmes: ceux qui envisagent les événements comme essentiellement dépendant de facteurs externes (chance, destin, etc.) et ceux qui les envisagent comme essentiellement dépendant de facteurs internes (liés à des caractéristiques et comportements personnels). La vulnérabilité face à des agents stressants serait d'ailleurs plus importante pour les individus fatalistes, percevant ce qui leur arrive comme provenant de facteurs externes. Pour Rosenstock et al. (1988), les échecs dans l'observance des prescriptions ou dans l'adoption de comportements de soins seraient également liés au fait que les individus évaluent mal l'impact réel de la maladie sur leur vie. Ces échecs pourraient enfin être liés au fait que les efforts à faire par le patient pour éviter de telles conséquences sont supérieurs au bénéfice direct du soin pour le patient (Home, James, Petrie, Weinman \& 
Vincent, 2000; Home, \& Weinman 1999; Bradley, 1994; Norman, 1995; Shiloh, Rashuk-Rosenthal \& Benyamini, 2002).

Enfin, la qualité de la relation thérapeutique avec le soignant est le facteur prépondérant dans l'observance. Le patient peut rechercher ou percevoir des éléments dans la relation avec le médecin qui vont le rassurer ou le soutenir psychologiquement. La qualité de cette relation clinique serait un élément fondamental dans la mise en place de comportements de soins. Hessen-Klemens et Lapinska (1984) ainsi que Squier et Jackson (1990) ont montré que plus le médecin encourage et soutient son patient plus celui-ci est observant dans la mise en œuvre des comportements de soins. La chaleur humaine et l'empathie seraient donc essentielles pour l'observance. De même pour Rosenstock et al. (1988), la qualité de la relation renforcerait la perception qu'a le patient d'un contrôle personnel sur l'évolution de la maladie et ses attentes positives quant aux bénéfices d'un comportement observant. L'interaction ayant lieu lors de la relation thérapeutique pourrait contribuer à un soutien psychoaffectif pour le patient, soutien apporté par le médecin lorsque celui-ci retire également une satisfaction personnelle de sa relation avec le patient (Squier, Jackson, 1990; Hessen-Klemens, Lapinska, 1984; Gao, Nau, Rosenbluth, Scott, Woodward, 2000).

\section{Asymétrie de la relation de soin, rationalisation des rôles et points de vue attendus}

A notre connaissance, aucun travail empirique ne s'est jusqu'alors intéressé à la façon dont les soignants eux-mêmes percevaient les interactions au sein de la triade soignant- patient-aidant naturel, notamment en ce qui concerne la prise en charge et l'évolution de la maladie.

Des travaux tentent pourtant d'analyser de façon générale la relation soignésoignant et de proposer des modèles interprétatifs de cette relation (Le Sommer-Péré, M., 2000). Un premier modèle est un modèle de relation pouvant être qualifié de «paternaliste» où le soignant, représenté dans une posture bienveillante, connaît et sait ce qui est bien pour le patient, du fait de son savoir et de son expertise médicale. Le patient y est représenté dans une posture passive de dépendance et d'attente vis-à-vis du soignant et du soin. Un second modèle plus récent se veut contractuel et soutient lidée du libre choix du patient, de son adhésion préalable au projet de soin et de son droit à linformation. Un dernier modèle, de relation pédagogique, rappelle que le malade est en situation de crise et que son état de souffrance altère temporairement la rationalité de sa décision et ses capacités de coopération. Le soignant aura la responsabilité de la conception du programme de soin et des conditions de sa mise en œuvre, mais devra les expliciter au patient tout au long du soin. En fait, quelque soit le modèle envisagé, l'objectif du soin est toujours la guérison et celle-ci passe nécessairement par l'aide du soignant et sa maîtrise des soins médicaux, représentant une asymétrie difficilement contournable dans la relation entre soignant et patient. Il est vraisemblable que les soignants élaborent leur représentation de l'évolution thérapeutique et de ses déterminants sur la base de cette représentation de la relation soigné-soignant dont l'objectif est la guérison par le biais de leur maîtrise des actions de soins à mettre en place. 
D'une façon générale, il est fréquent que les pratiques professionnelles déterminent largement les représentations que les sujets ont d'un objet (Abric, Flament, Guimelli, Mardellat, Morin \& Singery, 2001). Dans de nombreux secteurs d'activités, il est observé que les travailleurs sociaux rendent cohérente avec leur pratique l'analyse causale des situations auxquelles ils sont confrontés. Ainsi, dans le domaine de l'accidentologie routière, les différents protagonistes tendent à minimiser leur propre rôle dans la survenue des accidents et se centrent sur des déterminants justifiant leur position ou leur rôle professionnel (Kouabenan, 2002). Dans les activités de maintien de l'ordre, les policiers tendent à attribuer les comportements déviants à des déterminants internes et stables aux personnes qu'ils doivent interpeller (par exemple leur personnalité ou prédispositions déviantes). Les surveillants de prison, quant à eux, attribuent ces mêmes conduites déviantes à des facteurs essentiellement stables dans le temps, justifiant ainsi l'incarcération (facteurs qui sont alors aussi bien d'ordre interne qu'externe aux personnes les produisant ; Abdellaoui, Kouabenan \& Gilibert, 1998).

Dans le domaine de l'éducation enfin, les enseignants tendent à considérer que les performances scolaires sont majoritairement liées aux efforts des élèves (caractéristiques internes et contrôlables par ces derniers) et ce d'autant plus qu'ils les ont notés de façon polarisée ou qu'ils leur est demandé de rendre compte du niveau scolaire de l'élève (Matteucci \& Gosling, 2004 ; Gosling, 1994 ; 1995). Les explications des enseignants tendent aussi à être différentes en fonction de la valeur scolaire de l'élève : alors que les résultats d'un élève ayant un bon niveau sont attribués aux efforts quil aurait produits, ainsi qu'à la qualité de l'encadrement pédagogique, les résultats d'un mauvais élève sont essentiellement attribués à un manque d'efforts au niveau scolaire (à des éléments plus fréquemment internes, instables et spécifiques au domaine scolaire). Les explications évoquées permettent ainsi de justifier les pratiques évaluatives et la qualité de l'encadrement pédagogique.

Outre le fait que les causes envisagées varient en fonction du niveau scolaire de l'élève, il semble enfin que les enseignants s'attendent à des explications bien différentes de la part des bons et des mauvais élèves ; explications pouvant justifier la moindre performance de ces derniers. D'après les enseignants, le point de vue des mauvais élèves à la différence de celui des bons élèves, serait que les performances (bonnes ou mauvaises) sont imputables à des facteurs extérieurs ou incontrôlables par eux. Dans ces travaux basés sur un «paradigme d'identification », les sujets, les parents ou les enseignants, doivent envisager et rapporter le point de vue d'un bon et d'un mauvais élève (Dubois \& Beauvois, 1996; Beauvois, Gilibert, Pansu \& Abdellaoui, 1998; Dubois, 2003). Dans ce domaine, comme dans d'autres domaines de la perception sociale, il apparait que les personnes les moins valorisées socialement, ici les mauvais élèves, se voient attribuer les croyances externes qui sont peu valorisées socialement (que la valeur soit définie sur la base du statut professionnel, académique, scolaire ou de façon plus affective, Gilibert \& Cambon, 2003 ; Pansu, Tarquinio \& Gilibert, 2005). Rappelons qu'en matière d'attributions causales, les croyances les moins valorisées sont relatives à une détermination externe plutôt qu'interne de nos résultats, ou encore à des facteurs incontrôlables plutôt que contrôlables par soi-même. 
Etant donnée le caractère asymétrique de la relation de soin, on peut alors s'attendre à ce que les déterminants de l'état de santé soient décrits par les soignants de façon cohérente avec leur objectif de soin et leur maitrise du soin et de la prise en charge du malade. On peut ainsi s'attendre (hypothèses unilatérales) à ce que :

1/ les soignants se représentent les déterminants de l'état de santé comme : a) ayant un impact général sur la vie du patient, mais b) demeurant toutefois remédiables (le soin ayant pour finalité d'améliorer l'état de santé du patient), c'est-à-dire instables et susceptibles d'évoluer et c) contrôlables par l'aide d'autrui, mais a) n'étant pas contrôlables par le patient ni particulièrement lié à lui-même (l'état de santé étant supposé dépendre du soin prodigué).

2/ les soignants se représentent le point de vue du malade comme moins centré sur le problème (sous-estimant le fait que ces déterminants sont décisifs et remédiables) et plus passif (surestimant l'intervention d'éléments extérieurs et incontrôlables par luimême) ; justifiant ainsi leur préséance sur les soins. Non seulement le patient surestimerait des croyances qui apparaissent comme néfastes dans le cadre du HealthBelief Model (extemalité, incontrôlabilité) mais il sous-estimerait aussi des croyances utiles (généralité de l'impact des déterminants de l'état de santé, instabilité, contrôlabilité par autrui).

3/ lorsque l'état de santé s'est détérioré, les soignants devraient avoir une perception moins optimiste des déterminants de l'état de santé et surtout rapporter des points de vue plus fortement divergents de la part du malade et de sa famille : ceux-ci seraient perçus comme ayant des croyances externes et d'absence de contrôle, particulièrement prononcées dans les cas où la prise en charge s'est avérée difficile et l'évolution de la maladie la moins favorable (en accord avec les travaux sur la norme sociale d'internalité).

\section{Méthode}

\section{Population:}

La population était constituée de quarante-quatre soignants, dix hommes et trente-quatre femmes, infirmier(e)s, diplômé(e)s d'état depuis plusieurs années (infirmiers anesthésistes, puéricultrices, infirmiers, infirmiers psychiatriques, infirmiers de bloc opératoire, manipulateurs-radio), en formation pour devenir soit cadres de santé, puéricultrices ou anesthésistes. Leur âge moyen est de 31,5 ans, dont plusieurs années de service (en moyenne 8 ans).

\section{Procédure:}

La passation du questionnaire, lors de sessions de formation, était présentée comme relevant d'une recherche dont l'objectif était de mieux comprendre les déterminants des prises en charge thérapeutique et des comportements de soin. 


\section{Phase 1 : évocation de deux cas cliniques contrastés en termes d'évolution}

Après quelques informations signalétiques (spécialité, âge, expérience), il leur était demandé de se remémorer deux cas cliniques de leur domaine de spécialité qu'ils avaient suivis et qu'ils avaient bien connus, atteints de la même pathologie avec le même niveau initial de gravité. Pour l'un des patients, la prise en charge thérapeutique devait s'être plutôt bien passée avec une évolution favorable de la maladie et, pour l'autre, la prise en charge devait avoir été plus difficile avec une évolution défavorable de sa maladie. Ils devaient tout d'abord rapporter le nom de la pathologie et le degré de gravité initial des symptômes. Les pathologies rapportées couvrent un vaste ensemble de pathologies somatiques extrêmement variées (peu de pathologies psychiatriques ont été évoquées). Ils devaient également, et pour chacun des deux cas séparément, mentionner la durée de suivi. Le suivi de ces cas cliniques a duré en moyenne 202 jours, qu'il se soit bien ou mal passé, avec une très forte variabilité dans chaque cas du fait de la variété des pathologies (bien passé: $\mathrm{m}=202.7$ e-t.=539.6 vs. Mal passé: $\mathrm{m}=201.8$ et.=413.9).

\section{Phase 2 : point de vue des soignants sur les déterminants de l'état de santé et points de vue rapportés pour les patients et leurs familles}

Ils étaient avertis, qu'afin d'être exhaustifs dans la recherche des déterminants possibles de l'état de santé, qu'ils devraient non seulement donner leur propre point de vue sur les déterminants de l'état de santé des patients, mais qu'ils devraient également rapporter ce qu'était ou leur a semblé être le point de vue de ces patients et de leurs familles. Après avoir évoqué de façon qualitative les déterminants possibles de l'évolution de l'un de ces deux cas, il leur était demandé à partir d'échelles dimensionnelles de caractériser les éléments qu'ils estimaient être déterminants de cette évolution de la maladie du patient (évolution soit favorable ou soit défavorable, selon l'ordre de réponse des questionnaires aléatoirement distribués aux infirmiers). Ces échelles dimensionnelles sont basées sur les distinctions habituellement faites entre facteurs de causalité (Weiner, 1985; Fontayne, Martin-Krumm, Buton \& Heuzé, 2003) : 1/ le lieu d'origine de la cause interne ou externe au patient, 2/ la stabilité temporelle des causes (permanentes ou aléatoires), 3/ la généralité de leur impact dans la vie du patient, et 4/ la contrôlabilité de causes: contrôlables ou non par le patient. Une cinquième dimension relative à la contrôlabilité de ces causes par l'aide d'autrui a paru pertinente dans la mesure où l'étude porte sur une relation d'aide et de soin apporté par autrui.

Après avoir donné leur opinion sur les déterminants de l'évolution de la maladie, les soignants étaient invités à indiquer, sur un format identique, ce qui leur avait semblé être l'opinion du patient sur les déterminants de sa maladie, puis ce qui leur avait semblé être l'opinion de sa famille(2). La procédure de réponse était répétée alors pour le second cas clinique contrasté en terme d'évolution de la maladie (soit favorable soit défavorable). 


\section{Phase 3 : questions de vérification concernant les cas cliniques et l'évolution thérapeutique}

Dans un dernier temps les sujets complétaient un questionnaire de description clinique pour chacun de ces deux cas. Il permettait d'évaluer la gravité de la maladie, son évolution au cours de la prise en charge et l'impact du traitement thérapeutique (Clinical Global Impression Scale, voir partie « matériel ») ainsi que l'impact de la maladie sur le fonctionnement psychosocial du patient (Global Assessment of Functioning Scale).

\section{Matériel}

\section{Représentation des déterminants de l'état de santé et de leurs caractéristiques causales.}

Dans un premier temps, il était demandé aux soignants, en fonction de leur pratique clinique et leur expérience de la maladie, de mentionner par écrit des éléments qui leur avait parus déterminants de l'apparition des symptômes, de la consultation et de l'évolution favorable (vs. défavorable) de sa maladie pour chaque cas clinique(3).

Concernant plus particulièrement l'évolution (favorable vs. défavorable) du patient, il leur était alors demandé si ce qui avait déterminé cette évolution a) Etait liée à des éléments extérieurs à lui-même. b) Etait liée à lui, à sa personnalité, à sa façon d'être, c) Etait stable et peu susceptible d'évoluer dans le temps d) Etait aléatoire et susceptible d'évoluer dans le temps, e) Etait contrôlable, maîtrisable par lui, f) N'était pas contrôlable, n'est pas maîtrisable par lui, g) N'était susceptible d'affecter son comportement que dans peu de situations, h) Etait susceptible d'affecter son comportement dans d'autres situations de la vie, i) Etait contrôlable, maîtrisable grâce à l'aide apportée par autrui, j) N'était pas contrôlable, maîtrisable grâce à l'aide apportée par autrui. En face de chacune de ces affirmations, les sujets indiquaient leur degré d'accord (sur une échelle d'opinion de Likert en 7 points allant de «pas du tout » à « tout à fait » par la suite codée de -3 à +3 ). Ces réponses permettaient d'évaluer leur perception de l'origine de la maladie sur les cinq dimensions attributives retenues pour cette étude: le lieu d'origine de l'évolution thérapeutique, interne/externe au patient ; la contrôlabilité/incontrôlabilité des éléments déterminants par le patient ; la stabilité/ l'instabilité dans le temps de ces déterminants ; l'impact général/spécifique de ces déterminants sur le comportement du patient et enfin leur contrôlabilité/incontrôlabilité par l'aide d'autrui. Cette méthode de mesure est similaire à la version révisée de mesure des attributions causales permettant de d'investiguer les dimensions causales les plus pertinentes dans les théories de l'attribution (Causal Dimension Scale, adaptée en français par Fontayne, Martin-Krumm, Buton \& Heuzé, 2003). Conformément à celleci, nous traiterons de façon statistique la différence entre les deux items antagonistes pour chacune des cinq dimensions (e.g. : l'attribution au patient moins l'attribution aux éléments externes). 


\section{Questions de vérification concernant les cas cliniques et l'évolution thérapeutique}

Afin de vérifier que chaque cas correspondait bien à une évolution favorable ou défavorable de la maladie, il leur était demandé de produire une évaluation clinique de chaque patient après sa prise en charge par le biais de la Clinical Global Impression Scale en plusieurs échelles fréquemment utilisées dans le domaine médical (Guy, 1976)(4). Les valeurs de la première échelle (item 1) ont été comparées à une variante présentée en tout début de questionnaire pour situer la gravité des symptômes (la pathologie et les symptômes devait être identiques entre les deux cas ; le niveau de gravité initiale des symptômes $(\mathrm{m}=5.43)$ au moment de la prise en charge correspondant en moyenne à des cas se situant entre «manifestement » et « gravement malade »). Il apparait que les symptômes sont jugés tout aussi sévères en fin de traitement dans le cas d'évolution défavorable $(\mathrm{m}=5.16, \quad \mathrm{~F}(1,43)=1.2 \mathrm{~ns})$ mais qu'ils s'atténuent significativement en cas d'évolution favorable $(\mathrm{m}=4.59, \mathrm{~F}(1,43)=8.69 ; \mathrm{p}<.01)$. La seconde échelle, évaluant l'amélioration de l'état de santé, atteste d'une évolution positive dans le cas favorable $(\mathrm{m}=2.04$; «Fortement amélioré ») et d'une stagnation dans le cas défavorable $(\mathrm{m}=3.82$; «pas de changement », ces évolutions étant bien différentes; $\mathrm{F}(1,43)=32.0 ; \mathrm{p}<.0001)$. La différence entre les deux patients est confirmée par la troisième et dernière mesure de l'effet du traitement (CGI item3 : 3.98 vs. 8.24, $\mathrm{F}(1,4$ 1) $=27.1 ; \mathrm{p}<.0001$ 1) avec une «disparition importante des symptômes» rapportée pour le cas favorable alors qu'un effet seulement «minime » est rapporté pour le cas défavorable ( 1.50 vs. $2.52, \mathrm{~F}(1,4 \mathrm{l})=29.6 \mathrm{p}<.0001)$, sans qu'il n'y ait plus des effets secondaires rapportés pour le cas le plus défavorable ( 1.98 vs. $2.14, \mathrm{~F}(1,41)=0.8 \mathrm{~ns})$.

En toute fin de questionnaire, les soignants devaient évaluer l'impact de la maladie sur le fonctionnement du patient dans sa vie sociale, à l'issue de la prise en charge (Global Assessment of Functioning Scale (5). Cette mesure atteste également d'une différence d'évaluation en terme de fonctionnement psychosocial, entre les deux cas $(\mathrm{F}(1,42)=60.65 ; \mathrm{p}<.0001)$ avec une évaluation plutôt positive du cas favorable $\mathrm{m}=71.14 \%$ : «Quelques symptômes légers ou une certaine difficulté dans le fonctionnement social, professionnel ou scolaire mais fonctionne assez bien de façon générale et entretient plusieurs relations interpersonnelles positives » et une évaluation clairement négative pour le cas défavorable $\mathrm{m}=40.84 \%$ : «Existence d'une certaine altération de la communication - discours par moment inadapté - ou déficience majeure dans plusieurs domaines (le travail, l'école, les relations familiales, le jugement) ».

\section{Résultats}

Pour chacune des 5 dimensions attributives, la différence entre les deux items antagonistes a été traitée par analyse de variance selon un plan entièrement intrasujet, en fonction de «l'évolution» de l'état de santé (cas Favorable vs. cas Défavorable) et en fonction « des points de vue envisagés » sur l'origine de l'état de santé (le point de vue du Soignant luimême vs. le point de vue quil rapporte comme étant celui du Patient concerné vs. le point de vue quil rapporte comme étant celui de sa Famille). Les moyennes sont présentées dans le tableau 1. Une première lecture descriptive des moyennes (une différence de 1 point avec zéro, 
est significative à un seuil $p<.05$ ) indique que les éléments contribuant à l'état de santé sont globalement perçus comme ayant un impact général (affectant le comportement du patient dans d'autres situations de la vie), instables (susceptibles d'évoluer dans le temps) et clairement contrôlables par l'aide apportée par autrui, mais plutôt incontrôlables par le patient (pour cette dernière dimension $p<.10$ ).

Tableau 1: Moyennes et écart-types des valeurs des échelles dimensionnelles pour caractériser l'évolution thérapeutique (favorable ou non) en fonction des points de vue envisagés par les soignants (eux-mêmes, rapporté pour le patient, rapporté pour la famille).

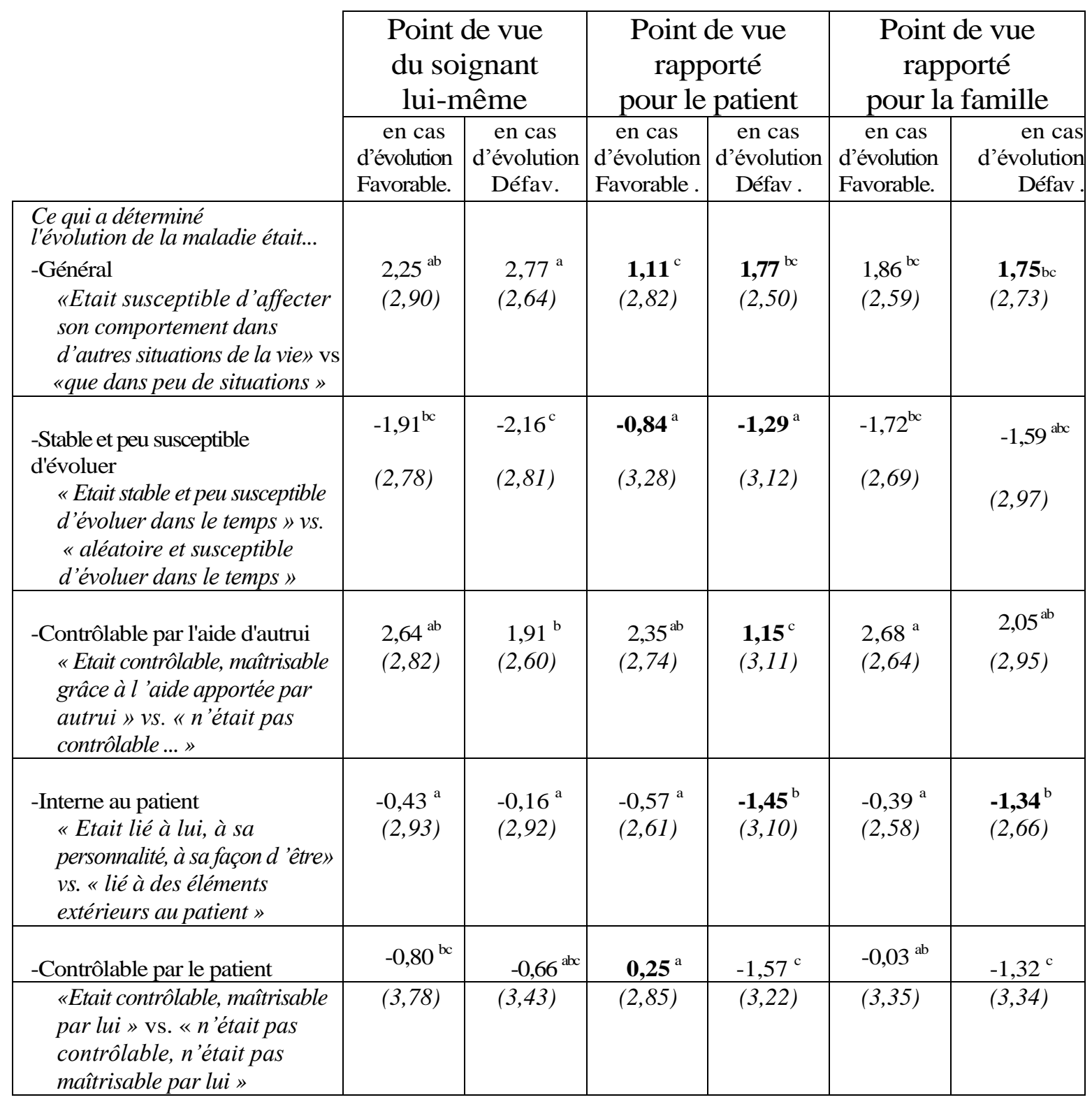

Note : Dans ce tableau, les moyennes ne portant en indice aucune lettre commune, sont statistiquement différentes à un seuil $p<.05$ (avec un test $F$ de Fischer, ou à un seuil $p$ bilatéral <.10, avec un test post-hoc LSD). Sont rapportées en gras les moyennes divergeant du point de vue des soignants pour le cas d'évolution concerné. 
Les résultats seront présentés en trois temps : 1/ les effets de « l'évolution » de l'état de santé sur la perception de ses déterminants, 2/ les effets des « points de vue envisagés » révélant des différences entre le point de vue du soignant et le point de vue rapporté par ce soignant pour le patient et sa famille et 3/ l'interaction de ces deux variables permettant de voir si les différences de points de vue sont dépendantes de l'évolution de l'état de santé.

\section{Perception des déterminants de l'état de santé en fonction de son évolution (favorable vs. défavorable)}

Le fait que l'état de santé ait évolué favorablement ou non amène des différences de description globale des déterminants de cette évolution. Les déterminants tendent à être décrits comme étant moins contrôlables par l'aide d'autrui lorsque l'évolution a été défavorable plutôt que favorable (1,70 vs. 2.56 vs. $0.03, \mathrm{~F}(1,43)=4.8$ $\mathrm{p}<.02)$, à être plus incontrôlables par le patient $(-1.18$ vs. $-0.19, \mathrm{~F}(1,43)=8.1 \mathrm{p}<.01)$ et d'autant moins liés au patient $(-0.98$ vs. $-0.46, F(1,43)=2.5 \mathrm{p}<.06)$. Néanmoins si l'on considère isolément les réponses des soignants, indépendamment des points de vue qu'ils rapportent pour le patient et sa famille, seule la différence de contrôlabilité par l'aide d'autrui tend en partie à être significative dans les réponses qu'ils endossent (2.64 vs. 1.91, $\mathrm{F}(1,43)=2.7 \mathrm{p}<.06)$.

\section{Différences entre les différents points de vue envisagés par le soignant (son propre point de vue, le point de vue du patient et celui de sa famille)}

$\mathrm{Si}$ on s'attache à étudier les différences globales de point de vue indépendamment de la qualité de l'évolution thérapeutique (favorable vs défavorable), les soignants rapportent des points de vue différents du leur pour le patient et sa famille en ce qui concerne quatre dimensions causales sur cinq $(\mathrm{F}(2,86)=6.2 \mathrm{p}<.01$ pour la généralité de l'impact des éléments déterminants l'évolution de la maladie, $\mathrm{F}(2,86)=3.83$ $\mathrm{p}<.02$ pour leur stabilité, $\mathrm{F}(2,86)=2.45 \mathrm{p}<.05$ pour la contrôlabilité par l'aide d'autrui et $\mathrm{F}(2,86)=2.24 \mathrm{p}<.06$ pour le lieu d'origine de ces éléments). La lecture du tableau 1 indique que les soignants s'attendent à des points de vue du patient et de sa famille qui sont moins polarisés que le leur mais allant néanmoins dans le même sens.

Si l'on fait abstraction de la qualité de l'évolution, peu de différences sont envisagées avec le point de vue de la famille qui occuperait une position intermédiaire entre le point de vue du patient et celui du soignant. Selon les soignants, la famille sousestimerait pourtant l'impact général des déterminants de santé comparativement à euxmêmes (1.81 vs. 2.51, LSD p-bilatéral<.05).

En comparaison avec leur point de vue, les soignants présente le patient comme moins centré sur la résolution de son problème de santé : il sous-estimerait l'impact des déterminants de santé (1.44 vs. 2.5 1, LSD p-bilatéral<.0 1), le fait qu'ils soient remédiables, c'est à dire susceptibles d'évoluer (-1.07 vs. -2.04, LSD pbilatéral<.01) et contrôlables par l'aide d'autrui (1.75 vs. 2.27, LSD p-bilatéral<. 10). 
Son point de vue serait également plus passif, dans la mesure où il sous-estimerait le fait que ces déterminants sont, au moins en partie, liés à lui-même (-1.01 vs.-0,30, LSD p-bilatéral<.05).

Aucune interaction significative n'apparaît pourtant pour la contrôlabilité de cette évolution par le patient lui-même. Cette variable est pourtant d'intérêt, tant au regard des travaux sur le Health Relief Model que du point de vue de la valeur socialement attribuée au contrôle personnel dans le cadre de la norme sociale d'internalité. On peut néanmoins s'attendre à une différence de point de vue dépendante le l'évolution (favorable ou non) de la maladie.

Interaction des deux variables : différences entre les points de vue envisagés en fonction de l'évolution (favorable vs. défavorable)

Des divergences apparaissent entre les trois points de vue envisagés en fonction de l'évolution favorable ou défavorable de la maladie, pour ce qui est de la contrôlabilité perçue de la maladie par le patient (interaction $\mathrm{F}(2,86)=2.9 \mathrm{p}<.05$ ) ainsi que du lieu d'origine de la maladie (interaction $\mathrm{F}(2,86)=2.5 \mathrm{p}<.05$ ). Les moyennes sont représentées dans les figures 1 et 2 . Aucune autre divergence significative entre les points de vue, liée à l'évolution de la maladie, n'est observée pour les autres dimensions.

Pour la contrôlabilité de la maladie, le point de vue du soignant lui-même ne semble pas changer que l'évolution ait été ou non favorable : dans les deux cas, le soignant est indécis et tendrait plutôt à penser que ce qui a déterminé l'évolution était plutôt incontrôlable par ces deux patients (6) (voir figure 1). Le soignant présente cette incontrôlabilité comme marquée dans l'esprit du patient et de la famille lorsque l'évolution a été défavorable plutôt que favorable (pour les patients : - 1.57 vs. 0.25, LSD p-bilatéral<.01 et pour les familles : -1.32 vs. -0.02, LSD p-bilatéral<.05). En cas d'évolution favorable, le patient est même présenté comme percevant sa maladie comme plus contrôlable que ne le faisait le soignant $(0,25$ vs. $-0.80, L S D$ bilatéral<.08). 


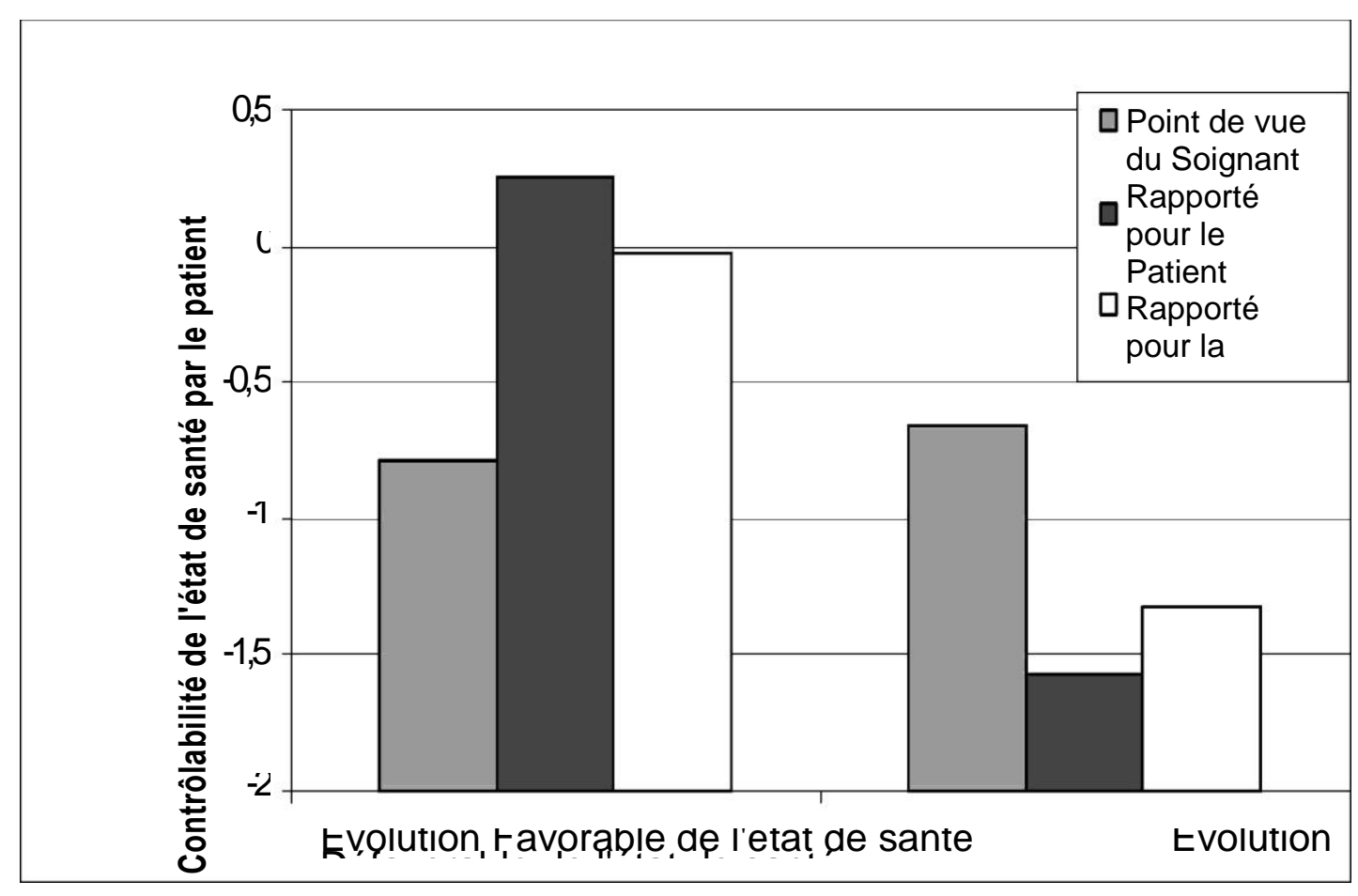

Figure 1

Moyennes pour la contrôlabilité des déterminants de l'état de santé en fonction des points de vue envisagés par les soignants (eux-mêmes, selon la famille, selon le Patient) en fonction de l'évolution (favorable vs. défavorable).

En ce qui concerne l'origine de la maladie (figure 2), le soignant estime qu'elle est tout autant liée au patient qu'à la situation extérieure, et ce quelque soit l'évolution. En cas d'évolution favorable, aucune différence n'apparait entre les trois points de vue envisagés. En revanche, en cas d'évolution défavorable, des points de vue différents du leur sont rapportés pour le patient et sa famille. Dans ce cas, les soignants présentent le point de vue du patient comme contestant le fait que cette évolution défavorable soit liée à la lui (-1.45 vs.-0.16, LSD p-bilatéral<.01). Selon eux, la famille, insisterait sur l'origine situationnelle de cette évolution défavorable, de façon plus prononcée qu'ils ne le feraient eux-mêmes (-1.34 vs.-0.16, LSD p-bilatéral<.01).

Patient et famille sont ainsi présentés comme plus externes dans leurs croyances lorsque l'évolution est défavorable que lorsqu'elle est favorable (pour les patients : -1.45 vs.- $0.57, L S D$ p-bilatéral $<.05$ et pour les familles : -1.34 vs. $-0.39, L S D$ p-bilatéral<.05). 


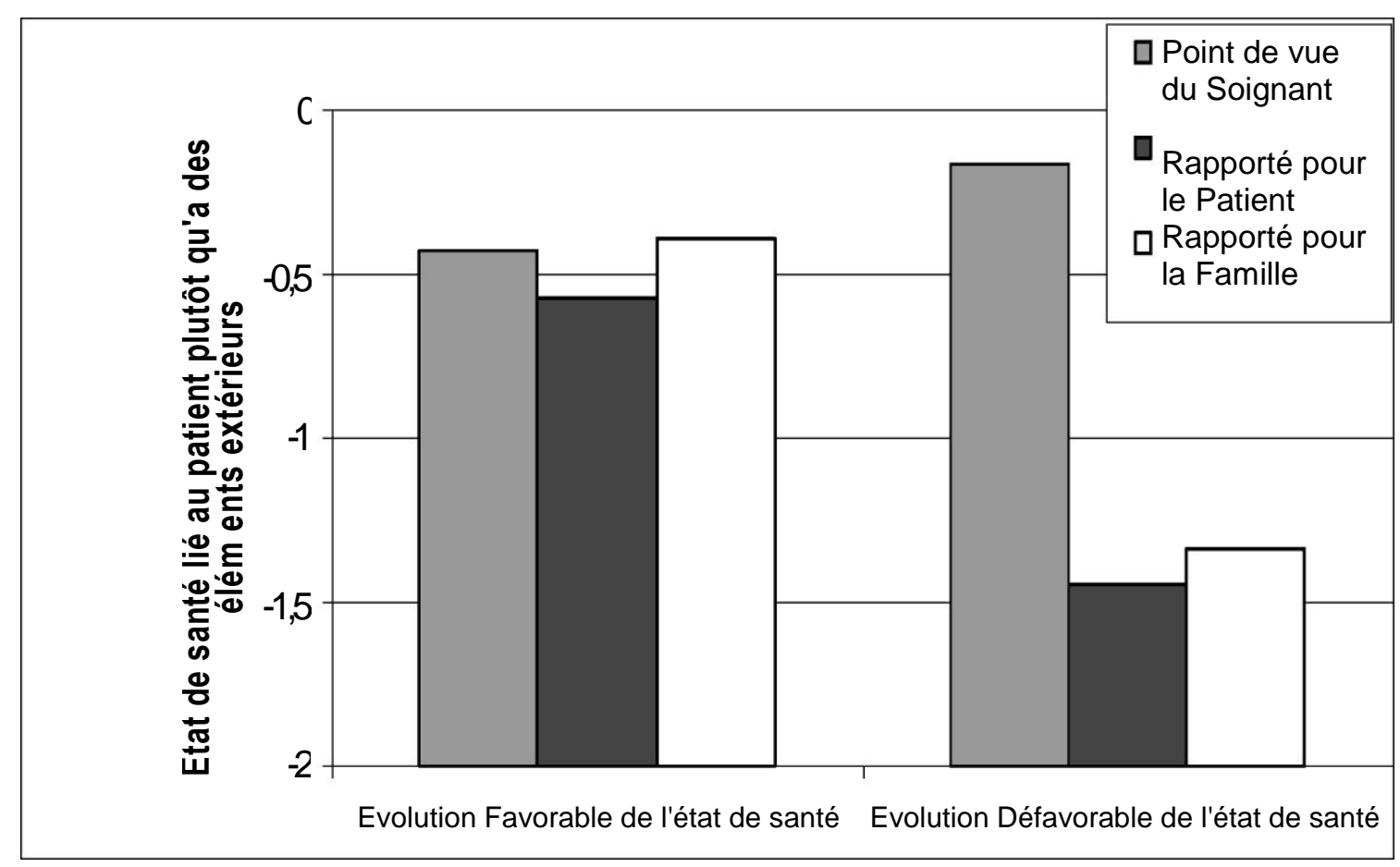

Figure 2

Moyennes pour l'origine des déterminants de l'état de santé en fonction des points de vue envisagés par les soignants (eux-mêmes, rapporté pour le patient, rapporté pour la famille) en fonction de l'évolution (favorable vs. défavorable).

\section{Conclusion}

Les résultats observés sont globalement conformes aux attentes que l'on pouvait avoir en se basant sur la nature asymétrique de la relation de soins. Trois éléments essentiels se dégagent et seront discutés successivement. Le premier élément concerne la perception que les soignants ont des déterminants de l'état de santé lors de la prise en charge. Le second concerne les points de vue qu'ils rapportent comme étant ceux des patients indépendamment de l'évolution de leur état de santé. Le dernier a trait aux points de vue rapportés comme étant ceux des patients et de ses aidants naturels lorsque la maladie suit une évolution défavorable.

\section{Une représentation de l'évolution de l'état de santé ancrée dans la nécessité du soin et l'état d'incapacité du patient}

Concernant tout d'abord la représentation des déterminants de l'état de santé, elle semble pour le moins cohérente avec la pratique de soin hospitalier. Rappelons que la relation soigné-soignant reste asymétrique du fait des connaissances médicales, de la maitrise du protocole de soin par les soignants et des places occupées par les différents acteurs (Le Sommer-Péré, 2000). Une représentation paternaliste et savante du soin continuerait d'influencer les attitudes des soignants et des malades en dépit de la nécessité de faire participer le patient à la décision de soin. Rappelons qu'elle dresse le 
portrait d'un malade passif, nécessitant un soin, en attente face à un soignant responsable et bienveillant, avec le risque d'une dépendance au discours et aux décisions médicales.

Conformément à cette représentation paternaliste, il a été observé que, du point de vue des soignants, le patient n'est pas en mesure de contrôler seul ce qui a déterminé sa maladie et son évolution, alors que ces déterminants auraient pourtant un impact général sur sa vie. Cette représentation est cohérente avec une vision du patient diminué, en attente de soins et n'étant pas en mesure de remédier par lui-même à son état de santé. Cela suggère aussi que, pour les soignants, le patient reste au second plan et ne ferait essentiellement que subir le soin et l'évolution de sa maladie. Par ailleurs, les soignants envisagent les éléments faisant évoluer l'état de santé comme remédiables : instables dans le temps et contrôlables par l'aide d'autrui. De façon cohérente avec leur objectif de soin, les soignants envisagent donc l'état de santé comme maîtrisable. Etant les initiateurs des soins, l'évolution de la maladie est de leur point de vue contrôlable par l'aide apportée et non par le par le patient, dont le rôle est relégué au second plan (le patient étant « pris en charge » par eux).

Cette représentation du suivi thérapeutique, par les soignants, justifie leur fonction de soin (la maladie ayant un impact général, étant susceptible d'évoluer mais contrôlable par l'aide apportée) ainsi que la position de chacun dans une relation définie comme asymétrique (la maladie étant contrôlable par l'aide apportée et non par le patient lui-même). Cette représentation de l'évolution de santé est néanmoins à relativiser au regard du contexte hospitalier. En effet, rappelons que les cas cliniques évoqués sont des cas le plus souvent graves et ayant nécessité une ou plusieurs hospitalisations. Ceci pourrait expliquer le peu de contrôle supposé du patient sur l'évolution de sa maladie et son impact, supposé important. Ces paramètres (pratique soignante avec ou sans prises en charges successives, représentation la contribution possible du patient au soin et de la relation avec lui, prise en charge ambulatoire) mériteraient d'être explorés dans une recherche ultérieure.

Enfin, de façon consistante avec les travaux sur les attributions causales de la maladie (Weiner, 1985), les soignants attribuent d'autant moins au patient un contrôle sur l'évolution la maladie, favorable ou non, que celle-ci relève d'une pathologie grave ou lorsqu'ils la considèrent comme invalidante pour le fonctionnement psychosocial du patient. Le fait, sans doute le plus troublant dans la représentation des déterminants de la prise en charge thérapeutique, est qu'elle ne semble quasiment pas varier en fonction de l'évolution de cet état de santé (amélioration effective ou stagnation). Les déterminants de l'état de santé tendent tout au plus à être perçus comme légèrement moins contrôlables par l'aide apportée en cas d'évolution défavorable. Aucune différence n'est observée concernant leur lieu d'origine, leur contrôlabilité par le patient, leur stabilité ou leur impact sur la vie du patient. Les recherches en matière d'analyse causale montrent pourtant de façon récurrente des effets liés à la désirabilité du fait expliqué, les explications divergeant facilement selon que les sujets aient à analyser une réussite ou un échec. On aurait ainsi pu s'attendre à ce que les déterminants soient perçus par les 
soignants eux-mêmes comme peu susceptibles d'évoluer, plus lourds de conséquences et moins contrôlables par le patient en cas d'évolution défavorable.

Il est vraisemblable que les infirmiers, de par leur fonction dans le cadre hospitalier, n'ont pas d'analyse causale à produire suite à l'évolution. Leur analyse causale de la maladie semble définie a priori sur la base du savoir médical, en fonction de la pathologie et de la gravité des symptômes (7). Il est aussi possible qu'invoquer des connaissances médicales et la technicité du rôle professionnel puisse permettre aux soignants de faire face aux affects pouvant être liés à l'issue défavorable de la maladie. Les éléments contribuant à l'évolution favorable ou non de la maladie, seraient alors à rechercher auprès d'autres soignants effectuant une action de prévention, un bilan du traitement ou qui suivraient régulièrement le patient : les médecins ou les infirmiers ayant des activités de secteur, de réseaux ou en libéral, voir les aidants naturels et les patients eux-mêmes.

\section{Des points de vues supposés convergents de la part des patients mais moins centrés sur la résolution du problème de santé}

Le second élément ressortant de ces observations concerne les points de vue qu'ils attendent globalement des patients. Certes les patients, en accord avec les soignants, reconnaîtraient l'impact des déterminants de la santé dans leur vie ainsi que leur caractère remédiable, c'est-à-dire aléatoire dans le temps et contrôlable par l'aide d'autrui («maladie-du-malade », Canguilhem, 1972 ; Pedinelli, 1987). Néanmoins, ils sous-estimeraient globalement l'importance de ces aspects. Au regard des travaux sur le Health Belief Model, on peut considérer que les soignants confèrent aux patients des croyances moins adaptées que les leurs. Les patients, moins que les soignants percevraient l'impact des éléments pouvant contribuer à leur état de santé ; ils sousestimeraient en partie l'aide qu'ils pourraient trouver dans leur environnement, notamment soignant. Cette représentation, en quelque sorte moins experte ou moins centrée sur le problème de santé, pourrait justifier le rôle des soignants ou leur préséance dans le soin à apporter : tout en reconnaissant la nécessité, le patient en serait moins convaincu, quelque soit d'ailleurs l'évolution de son état de santé.

Tout se passe donc comme si les soignants se représentaient le point de vue du patient comme proche du leur, le confortant en partie mais justifiant néanmoins leur fonction. On peut pourtant s'interroger sur la convergence effective de ces points de vue si les patients et les aidants naturels étaient réellement interrogés. En effet dans d'autres secteurs, comme celui de l'éducation, on sait que les points de vue peuvent diverger entre enseignants, élèves et parents (Gosling, 1992). Il est vraisemblable que les infirmiers passent peu de temps avec leur patient pour le consulter quant à la façon dont il perçoit l'origine de sa maladie : l'activité diagnostique revient au médecin ; lorsqu'ils disposent d'un peu de temps avec le patient c'est, vraisemblablement pour lui expliquer le point de vue médical sur l'origine de sa maladie et de son traitement (en accord avec un modèle de relation pédagogique plutôt que contractuel). 


\section{Un patient qui manifesterait son absence de contrôle en cas d'évolution défavorable de son état de santé}

L'élément le plus frappant concerne les représentations que les soignants rapportent comme étant celles des patients, concernant l'origine et la contrôlabilité des éléments faisant évoluer la maladie (mesures qui sont particulièrement pertinentes tant au regard du Health Belief Model, qu'au regard des travaux la valeur sociale des attributions causales). Si les soignants ne prennent pas position quant à savoir si ces éléments sont liés au patient ou à son environnement, ils s'attendent clairement à ce que le patient sous-estime ou minimise le fait que ces éléments puissent être liés à lui-même. Concernant les aidants naturels, ils s'attendent à ce que ceux-ci insistent sur les déterminants liés au contexte. De plus, cette différence avec leur propre point de vue n'est observée que lorsque l'évolution s'est avérée défavorable.

En fait, d'après les réponses données par les soignants, seuls les points de vue des patients et de leur famille seraient sensibles à l'évolution favorable ou non de la maladie. Dans les cas d'évolution défavorable, ces derniers manifesteraient une réaction « affective » : le patient préférait voir les raisons de l' échec thérapeutique comme n'étant pas liées à lui-même et sa famille tendrait à le dédouaner de la responsabilité de cet échec thérapeutique, en évoquant la présence de facteurs contextuels. De la même façon pour la contrôlabilité des déterminants de l'état de santé par le patient, les soignants s'attendent à ce que les patients et la famille présentent l'évolution comme étant particulièrement incontrôlable par le patient lui-même lorsqu'elle est défavorable. Pour ces deux mesures (internalité et contrôlabilité), les soignants semblent donc attribuer une attitude passive et fataliste au patient qui ne guérit pas. Dans les cas d'évolution défavorable, on suppose donc que le patient voit son évolution comme essentiellement déterminée par des éléments situationnels et incontrôlables par lui, sans que l'on puisse déterminer si, du point de vue des soignants, une telle perception par les patients serait la cause ou la conséquence de l'évolution de leur état de santé (ou à long terme les deux).

Du point de vue du système de relations entre soignant et patient, on peut s'interroger sur la façon dont les soignants réagissent face à des cas de patients prétendant n'avoir aucun contrôle sur l'évolution de leur maladie, notamment lorsqu'elle n'est pas particulièrement invalidante. Dans bien d'autres secteurs du travail, on sait par exemple que les employeurs, les enseignants ainsi que les travailleurs sociaux émettent des pronostics, des jugements ou prennent des décisions plus fréquemment défavorables vis à vis des personnes faisant état de croyances externes ou d'un faible sentiment de contrôle sur ce qui leur arrive (Desrumaux-Zagrodnicki \& Masclet 2001, Louche, Pansu \& Papet 2001 ; Dubois, 2003 ; Pansu, 2006) (8). On peut ainsi se demander quel peut être l'impact de cette attitude sur la pratique soignante et la prise en charge (en termes de soutien émotionnel apporté ou non, de qualité de suivi, de choix du traitement médical ou de participation des patients à la décision thérapeutique). 
Quoi qu'il en soit, la représentation de l'évolution de la prise en charge thérapeutique que les soignants ont donnée ici à voir, semble extrêmement normée par le savoir et leur rôle médical. Cette relation de soin asymétrique est sous tendue par une bienveillance à l'égard du patient et un objectif de guérison. Ainsi, les soignants demeurent extrêmement bienveillants en ne rejetant jamais la responsabilité de l'évolution de la maladie sur le patient (celle-ci n'étant ni spécifiquement liée au patient, ni contrôlable par lui et ce d'autant plus que la maladie est grave ; leur interprétation médicale ne variant d'ailleurs quasiment pas en fonction de l'évolution thérapeutique). Ils considèrent par ailleurs que ce qui détermine l'évolution de santé du patient, a un impact très général sur la vie du patient. En accord avec leur rôle de soignant, ils considèrent les déterminants de cette évolution comme remédiables et contrôlables par l'aide apportée au patient. De plus, selon eux, les patients en conviendraient sans en être aussi conscients. L'évolution défavorable de la maladie affecterait essentiellement les points de vue supposés du patient et de sa famille : le patient envisagerait ne pas pouvoir contrôler sa maladie ; sa famille partagerait son point de vue et mettrait en avant sur les circonstances de la maladie et de sa prise en charge.

Au niveau organisationnel, les évolutions qu'a connues la fonction infirmière semblent effectivement restreindre l'activité et l'analyse des infirmiers à l'application des soins. Les progrès techniques de la médecine ont réduit la durée des prises en charge ; leur nombre a augmenté (les pathologies devenant plus souvent chroniques que directement létales) alors que l'on fait face à difficulté à pourvoir les postes vacants (nombreux départs à la retraite accompagnés d'une diminution des vocations durables). La priorité alors donnée aux actes de soin (désormais quantifiés), plutôt qu'à l'échange avec les patients, peut avoir conditionné directement la perception que les infirmiers ont de l'état de santé de leurs patients (rôles nécessaires et prépondérants des soins et du soignant) ainsi que leur perception des différents points de vue.

\section{Note de fin}

(1) S'il est évident que les points de vue des patients et de ses aidants naturels sont pris en compte dans la pratique médicale afin d'établir les symptômes dont souffre le patient, la place qui leur est attribuée dans le soin est le plus souvent périphérique: la littérature existante sur le sujet mentionne majoritairement la prise en compte éventuelle de leur point de vue pour estimer limpact du traitement sur leur qualité de vie (Arnold, Ranchor, Sanderman, Kempen, Ormel \& Suurmeijer, 2004; Conroy, Guillemin \& Kaminsky, 2002; Parker, Baile, de Moor \& Cohen, 2003), l'impact de la prise en charge au domicile sur le fardeau, la charge ou l'épuisement (burden) des aidants naturels ou encore les informations fournies par la famille sur l'évolution de l'état de santé de patients dans l'impossibilité permanente ou temporaire de le faire (Andrieu, Balardy, Gillette-Guyonnet, Bocquet, Cantet, Albarède, Vellas, Grand, 2003 ; Gwyther, 1998; van Wijngaarden, Schene \& Koeter, 2004; Reine, Lançon, Avorio, Duplan, Siméoni, Aghababian, \& Auquier, 2004 ; Magliano, Fiorillo, De Rosa, Malangone, \& Maj, 2005).

(2) Seize questionnaires n'ont pas été pris en compte pour les analyses ici présentés, dans la mesure où certains infirmiers ont estimé ne pas être en mesure de rapporter le point de vue du patient et celui de sa famille (le plus souvent) pour l'un des deux cas requis. Pour les parties complétées leurs réponses ne différaient statistiquement pas des autres.

(3) D'un point de vue qualitatif, les réponses fournies par les soignants sont difficilement traitables. Concernant leur propre point de vue, elles sont essentiellement fondées 
sur l'état somatique (malformation congénitales, sur poids, tabac...) ou contextuelle (stress, travail difficile physiquement...). Le tableau clinique décrit est celui de la pathologie considérée et du traitement, assez indépendamment de son effet (le traitement lui-même et éventuellement l'état clinique antérieur plus complexe que prévu). Leur point de vue reste essentiellement centré sur le domaine médical même si l'influence de l'environnement familial est parfois évoquée (notamment dans les prises en charge pédiatriques). Le point de vue qu'ils supposent être celui des malades et des aidants naturels témoigne d'assez peu de différences avec leur propre point de vue (parfois avec une difficulté à énumérer autant d'éléments qualitatifs et à se décentrer de leur propre point de vue). L'absence a priori de divergence au niveau qualitatif se manifesterait notamment lorsque la prise en charge s'est bien passée: les malades et leur famille manifesteraient leur conscience des symptômes et viendrait dans les services de soin se faire aider (incapacité à respirer seul, le désir de guérir, le risque de perdre son autonomie...). En revanche, en ce qui concerne l'évolution défavorable, certains éléments seraient mis en avant et pas uniquement dans le registre d'un état clinique déjà difficile. Dans ce dernier cas, des facteurs familiaux sont rapportés pour les malades comme le divorce, le temps mis avant de se faire soigner ou la négligence de leur état somatique. La famille quant à elle, remettrait parfois en cause de la qualité et de l'efficacité des soins, invoquerait la fatalité liée souvent à l'état clinique déjà mauvais, remettrait en cause de la combativité du malade ou évoquerait sa propre difficulté à apporter une aide au malade (détresse, épuisement; absence de visite...).

(4) La Clinical Global Impression est constituée de 3 items indépendants utilisés dans presque toutes les études où on évalue une prise en charge médicale ou l'efficacité d'un médicament. Le principal avantage de ces trois items est leur simplicité d'emploi et le fait qu'ils soient adaptés à toutes les pathologies. Le premier item (mesure de la gravité du trouble actuel du patient) et le second (mesure de l'amélioration globale) sont quottés sur sept paliers de réponse. Le troisième item (mesure combinée de l'effet clinique et des effets secondaires) dispose de 16 réponses possibles. Cet item mesure à la fois le bénéfice thérapeutique (sur quatre paliers) et l'absence d'effets secondaires néfastes induits par le traitement (sur quatre paliers aussi). Ces trois items donnent lieu à trois mesures indépendantes et sont utilisés indépendamment les uns des autres. L'intérêt d'utiliser un tel outil est double. En début de questionnaire l'item 1 CGI, permettait aux soignants de situer le degré de gravité; le niveau de gravité devant être explicitement identique au départ dans le choix de leurs deux patients, seule l'évolution devant différer (évolution également vérifiable par les items 2 et 3 ).

(5) La Global Assessment of Functioning Scale (GAF) est une échelle d'origine psychiatrique (American Psychiatric Association) qui évalue le fonctionnement psychologique, social et professionnel de la personne (atteinte d'une pathologie psychiatrique dans sa version initiale). Il est demandé au praticien effectuant le suivi du patient d'évaluer le fonctionnement psychologique, social et professionnel global de celui-ci sur une échelle de 1 à $100 \%$. Des indicateurs clairs et concis sont offerts pour chaque tranche de 10 points de l'échelle. La GAF constitue le résultat opérationnel de l'axe V de l'évaluation multiaxiale du DSM-IV. Il s'agit d'une forme légèrement modifiée du Global Assessment Scale et peut être considérée comme fiable. Afin de la rendre plus facilement utilisable dans des situations cliniques non psychiatriques, toutes les références à un trouble mental ont été retirées ou remplacer par une référence simple : la maladie ». La forme initiale, pour le reste, a été conservée. La G.A.F. est pertinente ici car elle donne une vision complémentaire à la CGI de l'évolution des deux patients en élargissant l'évaluation des seules conséquences somatiques de la maladie au fonctionnement social du sujet.

(6) Indépendamment de l'évolution de la maladie (favorable ou non), la gravité initiale des symptômes semble être un facteur prédictif de l'incontrôlabilité des déterminants de santé par le patient. En effet les soignants considèrent que la maladie était d'autant moins contrôlable par le patient que ses symptômes étaient graves (pour le cas d'évolution favorable : $\mathrm{R}=-.39$ avec la 
contrôlabilité et $\mathrm{R}=+.37$ avec l'incontrôlabilité ; de même globalement pour l'évolution défavorable : $\mathrm{R}=-.31$ et $\mathrm{R}=0.13 \mathrm{~ns}$ ). De la même façon, ils considèrent que la maladie était d'autant moins contrôlable par le patient que son fonctionnement psychosocial est altéré (mesuré par la G.A.F. ; pour le cas d'évolution favorable $\mathrm{R}=+.42$ et $\mathrm{R}=.-.33$; pour le cas défavorable $\mathrm{R}=+.39$ et $\mathrm{R}=-0.18 \mathrm{~ns})$.

(7) Les infirmiers doivent avant tout suivre la bonne application du traitement prescrit par le médecin. Le diagnostic d'une telle évolution de santé et du bénéfice des traitements choisis, ne relève a priori pas de leur rôle, mais de celui d'un médecin référent. On peut pourtant s'inquiéter du fait que les infirmiers hospitaliers ne puissent différencier les déterminants d'une évolution effectivement défavorable et de ceux d'une évolution favorable, pour anticiper une telle évolution dans leur soin.

(8) Au-delà des choix alors à faire dans le traitement (que les soignants peuvent aisément contrôler par l'application strict d'un protocole) on peut se demander si, face à l'attitude de tels patients, leur satisfaction et la qualité de la relation n'est pas altérée de façon suffisamment perceptible par le patient pour amener ces derniers, au travers d'un cercle vicieux, à une moindre observance et une moindre croyance en l'effet du traitement (Hessen-Klemens \& Lapinska, 1984 ; Squier \& Jackson, 1990).

\section{Références}

Beauvois, J.-L., Gilibert, D., Pansu, P., Abdelhaoui, S. (1998). Internality attribution and intergroup relations. European Journal of Social Psychology, 28, 123 -140.

Andrieu, S., Balardy, L., Gillette-Guyonnet, S., Bocquet, H., Cantet, C., Albarède, J-L., Vellas, B., Grand, A., le groupe REAL.FR. (2003). Charge ressentie par les aidants informels de patients atteints de la maladie d'Alzheimer au sein de l'étude REAL.FR: méthode de mesure et facteurs associés. La revue de Médecine interne, 24, 351 s-359s.

Arnold, R., Ranchor, AV., Sanderman, R., Kempen, GI., Ormel, J., Suurmeijer, TP. (2004). The relative contribution of domains of quality of life to overall quality of life for different chronic diseases. Qual Life Res., 13, 883-96.

Benyamini, Y., Leventhal, H., Leventhal, EA. (2004). Self-rated oral health as an independent predictor of self-rated general health, self esteem and life satisfaction. In Social Science : Medicine, 59, 1109-1116.

Bradley, C. (1994). Measures of perceived control in diabetes. In Handbook of Psychology of Diabetes. London: Harwood Academic Publishers.

Bradley, C. (1995). Health beliefs and knowledge of patients and doctors in clinical practice and research. Patient Education and Counseling, 26, 99-106.

Canguilhem, G. (1972). Le normal et le pathologique. Paris : PUF.

Conroy, T., Guillemin, F., Kaminsky, MC. (2002). Mesure de la qualité de vie des patients atteints de cancer colorectal métastatique : techniques et principaux résultats. La revue de médecine interne, 23, 703-716.

Desrumaux-Zagrodnicki, P., Masclet, G. (2001). Stratégies d'explications internes et externes en fonction du contexte de chomage ou de recrutement, du niveau professionnel et du statut social. Bulletin de Psychologie, 54, 295-305.

Dew, MA., Goyocoolea, J., Harris, RC., Lee, A., Zomak, R., Dunbar-Jacob, J., Rotondi, A. (2004). An internet-based intervention to improve psychosocial outcomes in heart transplant recipients and family caregivers: development and evaluation. J Heart Lung Transplant, 23, 745-58. 
Dubois, N. (2003). A sociocognitive approach to social norms. London: Routledge

Daniel Gilibert et Ingrid Banovic / PTO - vol $14-n^{\circ} 1$

Dubois, N., Beauvois, J.-L. (1996). Internality, academic status and intergroup attributions. European Journal of Psychology of Education, 11, 329-341.

Elbogen, EB., Swanson, JW., Swartz, MS. (2003). Effects of legal mechanisms on perceived coercicion and treatment adherence among persons with severe mental illness. J Nerv Ment Dis., 191, 629-37.

Elder, JP., Ayala, G., Harris, S. (1999). Theories and intervention approaches to health-behavior change in primary care. Am J Prev Med, 17, 275-284.

Fischer, G-N., Tarquinio, C. (2006). Les concepts fondamentaux de la psychologie de la santé. Paris: Dunod.

Fontayne, P., Martin-Krumm, C., Buton, F., Heuzé, J.-F. (2003). Validation française de la version révisée de l'échelle de mesure des attributions causales (CDSII). Cahiers Internationaux de Psychologie Sociale, 58, 59-72.

Gao, X, Nau, DP., Rosenbluth, SA., Scott, V., Woodward, C. (2000). The relationship of disease severity, health beliefs and medication adherence among HIV patients. AIDS Care, 12, 387-98.

Gilibert, D., Cambon, L. (2003). Paradigms of the sociocognitive approach. In N., Dubois (Ed.), A sociocognitive approach to social norms, (pp 38-69), London: Routledge.

Goldring, A., Taylor, S., Kemeny, M., Anton, P. (2002). Impact of health beliefs, quality of life, and the physician-patient relationship on the treatment intentions of inflammatory bowel disease patients. Health Psychology, 21, 219-228.

Gosling, P. (1994). The attribution of success and faillure: the subject/object contrast. European Journal of Psychology of Education, 9, 69-83.

Gosling, P. (1995). Description vs. Explanation and academic evaluation. European Journal of Psychology of Education, 10, 41-59.

Graham, D., Kunik, M., Doody, R., Snow, A. (2005). Self-reported awareness of performance in dementia. in Cognitive Brain Research, 2005, in press. Available online at www.sciencedirect.com.

Guy W. (1976) Early Clinical Drug Evaluation (ECDEU) assesment manual for psychopharmacology. National Institute of Mental Health, Rockville.

Gwyther, LP. (1998). Social issues of the Alzheimer's patients and family. in The American Journal of Medecine, 104, 17S-21S.

Louche, C., Pansu, P., Papet, J. (2001). Normes de jugement et appréciation du personnel. Bulletin de Psychologie, 54, 369-374.

Hessen-Klemens, I., Lapinska, E. (1984). Doctor-patient interaction, patients' health behavior and effects of treatment. Social Science and Medicine, 19, 9-18.

Horne, R., James, D., Petrie, K., Weinman, J., Vincent, R. (2000). Patient's interpretation of symptoms as a cause of delay in reaching hospital during acute myocardial infarction. Heart, 83, 388-93.

Horne, R., Weinman, J. (1999). Patient's beliefs about prescribed medicines and their role in adherence to treatment in chronic physical illness. Journal of Psychosomatic Research, 47, 555-567.

Jakson, J., Kincey, J., Fiddler, M., Creed, F., Tomeson, B. (2004). Differences between outpatients with physical disease and those with medically unexplained symptoms with respect to patient satisfaction, emotional distress and illness perception. Br J Health Psychol., 9, 433-46.

Kouabenan, D.R. (2002). Occupation, driving experience and risk and accident perception. Journal of Risk Research, 51, 49-68. 
Magliano, L., Fiorillo, A., De Rosa, C., Malangone, C., Maj, M., the National Mental Health Project Working Group. (2005). Family burden in long-term diseases: a comparative study in schizophrenia vs. Physical disorders. Social Science \& Medicine, 61, 313-322.

Le Sommer-Péré, M. (2000). Relation soigné-soignant: quels modèles de référence? Perspective soignante, 9, 67-78.

Matteucci, M-C., Gosling, P. (2004). Italian and French teachers faced with pupil's Academic Failure: The "Norm of Effort". European Journal of Psychology of Education, 19, 147166.

Norman, P. (1995). Health locus of control and health behavior : an investigation into the role of health value and behaviour-specific efficacy beliefs. Personn. Individ. Diff., 18, 213218.

Parker, PA., Baile, WF., de Moor, C., Cohen, L. (2003). Psychosocial and demographic predictors of quality of life in large sample of cancer patients. Psychooncology, 12, 183-93.

Pedinelli, J-L. (1987). Le «travail de la maladie ». Psychologie Médicale, 19, 1047-1052.

Pibernik-Okanovic, M., Prasek, M., Poljicanin-Filipovic, T., Pavlic-Renar, I., Metelko, Z. (2004). Effects of an empowerment-based psychosocial intervention of quality of life and metabolic control in type 2 diabetic patients. Patient Educ Couns., 52, 193-9.

Politis, C. (1998). The psychosocial impact of chronic illness. In Ann N Y Acad Sci, 30, 349-54.

Pucheu, S., Consoli, S., D’Auzac, C., Français, P., Issad, B. (2004). Do health causal attributions and coping strategies act as moderators of quality of life in peritoneal dialysis patients? In Journal of Psychosomatic Research, 56, 317-322.

Reine, G., Lançon, C., Avorio, A., Duplan, S., Siméoni, MC., Aghababian, V., Auquier, P. (2004). Comment mesurer la charge des aidants naturels de personnes souffrant de schizophrénie ? Ann Méd Psychol, 162, 453-61.

Rosenstock, I.M. (1974). Historical origins of the health belief model. Health Education Monographs, 2, 354-386.

Rosenstock, I.M., Strecher, V.J., Becker, M.H. (1988). Social learning theory and the health belief model. Health Education Quarterly, 15, 175-183.

Shiloh, S., Rashuk-Rosenthal, D., Benyamini, Y. (2002). Illness causal attributions : an exploratory study of their structure and association with other illness coginitions and perceptions of control. Journal of Behavioral Medicine, 25, 373 -3 94.

Squier, D.J., Jackson, T.C. (1990). A model of empathic understanding and adherence to treatment regimens in practionner-patient relationships. Social Science and Medicine, 30, 325-339.

Van Wijngaarden, B., Schene, A., Koeter, M. (2004). Family caregiving in depression: impact on cargivers' daily life, distress and help seeking. Journal of Affective Disorders, 81, 211-22

Weiner, B. (1985). An attributional theory of achievment motivation and emotion. Psychological Review, 92, 548-573 